\title{
A program to further integrate mental health into primary care: lessons learned from a pilot trial in Tunisia
}

\author{
Jessica Spagnolo ${ }^{1}$, François Champagne ${ }^{1}$, Nicole Leduc ${ }^{2}$, Wahid Melki ${ }^{3}$, Nesrine Bram4, Imen Guesmi ${ }^{5}$, Michèle Rivard ${ }^{2}$, \\ Saida Bannour ${ }^{6}$, Leila Bouabid ${ }^{7}$, Sana Ben Hadj Hassine Ganzoui ${ }^{8}$, Ben Mhenni Mongi ${ }^{9}$, Ali Riahi ${ }^{10}$, Zeineb Saoud ${ }^{11}$, \\ Elhem Zine ${ }^{12}$, Myra Piat ${ }^{13}$, Marc Laporta $^{14}$, Fatma Charfi ${ }^{4}$ \\ 1 School of Public Health, Institut de recherche en santé publique de l'Université de Montréal (IRSPUM), Québec, Canada, ${ }^{2}$ School of Public Health, \\ Université de Montréal, Québec, Canada, ${ }^{3}$ Hôpital Razi, Service de Psychiatrie "D", Manouba, Tunisia, ${ }^{4}$ Université de Tunis El-Manar, Tunis, Tunisia, 5 \\ Centre médico-scolaire et universitaire de Manouba, Tunisia, ${ }^{6}$ Centre de soins de base Douar Hicher, Manouba, Tunisia, ${ }^{7}$ L'observatoire national des \\ maladies nouvelles et émergentes, Tunis, Tunisia, ${ }^{8}$ URR Ariana, Tunisia, ${ }^{9}$ Dispensaire Den Den, Manouba, Tunisia, ${ }^{10}$ Centre de soins de base \\ Ettadhamen, Ariana, Tunisia, ${ }^{11}$ Centre de soins de base Boumhel, Direction Régionale de la Santé de Ben Arous, Tunisia, ${ }^{12}$ Direction régionale de la \\ santé de Tunis, Centre de soins de base Taib Mhiri, Tunisia, ${ }^{13}$ Douglas Mental Health University Institute, Montréal, Québec, Canada, ${ }^{14}$ McGill \\ University, Montréal, Québec, Canada \\ Keywords: global health \\ https://doi.org/10.29392/joghr.3.e2019022
}

\section{Journal of Global Health Reports}

Vol. 3, 2019

\section{Background}

Tunisia is a lower-middle-income country located in North Africa. Since the 2010-2011 Revolution, a campaign of civil resistance to protest high levels of youth unemployment, difficult living conditions, and government corruption, a rise in mental health problems, substance use disorders, and suicide attempts/deaths has been recorded. To address untreated mental health symptoms, a mental health training program was offered to primary care physicians (PCPs) working in the Greater Tunis area of Tunisia, a collaboration between members of the Tunisian Ministry of Health, the School of Public Health at the Université de Montréal (Québec, Canada), the World Health Organization (WHO) office in Tunisia, and the Montréal WHO-PAHO Collaborating Center (CC) for Research and Training in Mental Health (Québec, Canada).

\section{Program description}

The training was based on the Mental Health Gap Action Programme (mhGAP) Intervention Guide (IG), a program developed by the WHO to help further develop the mental health competencies of non-specialists working in non-specialized settings. Our team adapted the $m h G A P-I G$ training to the primary care realities of the Greater Tunis area, offered the training program to PCPs between February and April 2016, and evaluated the program using a randomized controlled trial and implementation analysis.

\section{Discussion}

The adaptation, implementation, and evaluation of the training program equipped our team with important lessons learned, supported by evidence in the field of Global Mental Health. First, developing partnerships helped create a feasible program that met the practical and research needs of the country. Second, benefitting from political commitment to mental health facilitated the development of partnerships, the implementation of the training program, and the training's accompanying evaluation. Third, piloting the program helped identify challenges attributed to the training program and its implementation, the mental health care system, and the research tools, information that may be used to "build back better." Last, sharing research findings collaboratively helped ensure their validity and encouraged greater knowledge uptake.

\section{Conclusion}

We hope that sharing such lessons learned will aid other countries with similar profiles to develop and/or adapt, implement, and evaluate programs that target untreated mental health symptoms in primary and community-based settings and hence address priorities in Global Mental Health. 
Tunisia is a lower-middle-income country ${ }^{1}$ located in North Africa. Since the 2010-2011 Revolution, a campaign of civil resistance to protest high levels of youth unemployment, political repression, government corruption, and difficult living conditions, ${ }^{2}$ a rise in mental health problems, substance use disorders, and suicide attempts/deaths has been recorded. ${ }^{3-9}$ Through "the societal dialogue," a participatory process that aimed to understand the health concerns of Tunisian citizens and create possible health reform tracks that would aid decision-makers in improving the health of all, accessing mental health care was recognized as a key challenge. ${ }^{10}$ Commitment to improving access to needed mental health services was endorsed by the Tunisian Ministry of Health, particularly by the development of the 2013 Tunisian National Strategy for the Promotion of Mental Health ${ }^{6}$ and the creation of the Committee for Mental Health Promotion in 2015. Underlining the urgency of this commitment is also Tunisia's location within the Eastern Mediterranean Region (EMR), one of the World Health Organization (WHO) regions with the least number of countries to have produced a mental health plan or strategy ${ }^{11}$ and with one of the highest rates of mental disorder burden compared to the global average. ${ }^{12}$

Despite the Ministry's commitment to further the transition from institutional to community-based mental health care ${ }^{6}$ challenges to mental health care offered in primary care settings continue to abound. First, personnel trained in effective mental health care are lacking in number: 1) there are not enough mental health nurses and psychosocial care providers to meet current need ${ }^{13}$; and 2) while primary care physicians (PCPs) see patients consulting for mental health problems in primary care, studies highlight their limited competencies in detecting, treating, and managing mental illness. ${ }^{6,14-16}$ Second, while the Ministry has adopted the 2013 Tunisian National Strategy for the Promotion of Mental Health, some barriers continue to challenge the treatment and management of mental health conditions in primary and community-based settings: 1) substance use disorders are heavily stigmatized in Tunisia $6,17,18 ; 2$ ) restrictions placed upon PCPs related to the prescription of psychotropic medications ${ }^{19}$; and 3 ) the continued allocation of most of the funding for mental health (and, therefore, resources) to specialized care. $6,17,18$

Feasible and scalable ways to address the rise of untreated mental health symptoms in primary care settings is therefore a priority in Tunisia and in other low- and middleincome countries (LMICs) facing similar issues. ${ }^{20-22}$ Given the involvement of PCPs in mental health care in Tunisia, albeit with limited competencies, ${ }^{6,14-16}$ a mental health training program was offered to these non-specialists. Specifically, a training program based on the Mental Health Gap Action Programme (mhGAP) Intervention Guide (IG) (version 1.0), ${ }^{23}$ developed by the WHO, was offered to PCPs working in the Greater Tunis area of Tunisia between February and April 2016, and evaluated between January 2016 and September 2017. The training program's implementation and evaluation were part of a pilot project undertaken collaboratively between members of the Ministry of Health in Tunisia, the School of Public Health at the Universite de Montréal (Québec, Canada), the WHO office in Tunisia, and the Montréal WHO-PAHO Collaborating Center (CC) for Research and Training in Mental Health (Québec, Canada).

The $m h G A P-I G$ is a training included under the $m h G A P$ umbrella, an evidence-based program that aims to help build system capacity in LMICs by further developing and integrating mental health into primary care and community-based settings. ${ }^{24,25}$ The $m h G A P-I G$ training, currently in its second version, ${ }^{26}$ is used to help train non-specialists working in non-specialized settings in effective mental health care for what the WHO considers priority mental, neurological, and substance use disorders in LMICs. These include: depression, psychosis, epilepsy/seizures, developmental disorders, behavioural disorders, dementia, alcohol use disorders, drug use disorders, and self-harm/suicide. ${ }^{23,26}$ The guide is unique. First, the $m h G A P-I G$ was developed through a rigorous process. A systematic review of evidence available in mental health (e.g. detection, treatment, and management) was conducted, extracting data on interventions that have been proven effective. ${ }^{27}$ The $m h$ $G A P-I G$ presents these interventions (i.e. "what to do") using easy-to-follow diagrams. ${ }^{23,26}$ Second, the $m h G A P-I G$ was developed through international participatory processes. Specifically, the guide was developed by including expert opinions from researchers, decision-makers, and healthcare professionals. ${ }^{27,28}$ Third, the guide is updated every couple of years to include the latest evidence on mental health care delivery in LMICs specifically, as well as extensive feedback from experts who have used its previous versions. ${ }^{26,28}$ Last, the $m h G A P-I G$ is accompanied by training and evaluation tools to facilitate implementation and research. These include: facilitator guides, trainee guides, PowerPoint presentations, a contextualization guide to help adapt the training material and content to local healthcare realities, knowledge questionnaires, and supervision sheets. $^{28}$

Since its launch in 2010, the $m h G A P-I G$ training has been utilized in over a hundred countries. ${ }^{29,30}$ Given that it is a standard training program, the WHO suggests its adaptation before implementation. The next section of the paper gives a brief overview of the training program's adaptation to and implementation in the Greater Tunis area of Tunisia.

\section{PROGRAM DESCRIPTION}

Members of the Tunisian Ministry of Health (WM and FC) chose specific mhGAP-IG training modules ${ }^{23}$ considered priorities in the country. These included: general principles of care, depression, psychosis, suicide/self-harm, and substance use disorders (i.e. alcohol and drug use). Using the mhGAP-IG's accompanying Adaptation Guide, these modules were adapted to meet the Greater Tunis area's local primary healthcare realities in consultation with members of the Tunisian Ministry of Health, three Tunisian psychiatrists ("trainers"), and seven physicians responsible for continuing medical education in the Greater Tunis area ("tutors"). ${ }^{17}$ Tutors, well-versed in mental health detection, treatment, and management, were assigned to help trainees during and after training. They also assisted trainers during training sessions.

The training was conducted over six weeks for a total of 19 hours. The first five weeks consisted of general lec- 
tures, role plays, and group discussions on the chosen modules, totaling 17 hours. The last training session consisted of a two-hour support session animated by trainer-psychiatrists. This session allowed trainees to present challenging mental health cases and perform further role plays. Figure 1 highlights components of the training's adaptation and implementation in the Greater Tunis area of Tunisia.

The training program was evaluated in two ways. First, using a randomized controlled trial, our team assessed the training program's impact on PCPs' mental health knowledge, attitudes, self-efficacy, and self-reported practice (i.e. the importance allocated to mental health care per week and the number of referrals to specialized services done per week). These competencies and practice characteristics are listed as "outputs" in Figure 1. Second, using implementation analysis, our team explored how contextual factors might influence the program's implementation (i.e. through the adaptation of the training program to local primary healthcare realities of the Greater Tunis area ${ }^{17}$ ) and might interact with the program to influence its expected outcomes. ${ }^{19}$

\section{OBJECTIVE}

In this paper, we share the lessons learned from our program that focused on further integrating mental health into primary care in Tunisia by adapting, implementing, and evaluating a training program based on the $m h G A P-I G$ (version 1.0$)^{23}$ in the Greater Tunis area. Such lessons are supported by relevant literature in the field of Global Mental Health. We hope that our experiences may be useful to other LMICs in their quest to address untreated mental health symptoms with similar programs in primary or communitybased settings.

\section{DISCUSSION}

\section{LESSON 1: DEVELOPING PARTNERSHIPS}

Partnerships are relationships between stakeholder groups with different skills and expertise but collaboratively working together to accomplish a goal. ${ }^{31}$ In the case of our program, relationships with the following partners were developed: a research institution (the School of Public Health at the Université de Montréal), the political realm (the Tunisian Ministry of Health), the medical field (members of the Tunisian Ministry of Health who closely collaborated, through their affiliation with Hôpital Razi and Hôpital Mongi-Slim, with three Tunisian psychiatrists and seven PCPs in charge of continuing medical education in the Greater Tunis area), and international organizations (the WHO office in Tunisia and the Montréal WHO-PAHO CC for Training and Research in Mental Health). Of note, these partnerships also constitute ties between a high-income country (HIC) (i.e. Canada) and an LMIC (i.e. Tunisia).

A priority in Global Mental Health is to create partnerships in order to generate information that establishes "the health needs in a given setting, to propose culturally apt and cost-effective individual and collective interventions, to investigate their implementation, and to explore the obstacles that prevent recommended strategies from being implemented". ${ }^{32}$ However, the traditional position of research institutions in HICs-that is, as producers and gatekeepers of knowledge, following their own research agendas independently of those of key stakeholders where research is to be conducted $^{33}$-fails to address this priority in the field of Global Mental Health. ${ }^{34}$ Instead, partnerships must ensure that needs are adequately identified, articulated, and addressed, specifically by stakeholders with vested interest in them. ${ }^{35}$ Partnerships must also ensure methodological aspects of research are developed to ensure their feasibility within local contexts. ${ }^{36}$

Input from members of the Ministry of Health guided our program. Based on their involvement in the development and launch of the 2013 Tunisian National Strategy for the Promotion of Mental Health, ${ }^{6}$ they highlighted practical and research needs to be addressed in collaboration with our research team, using each stakeholder group's strengths and skills. For example, while mental health training programs have been offered to PCPs in Tunisia, these were not offered as part of a systematic national program, such as under the leadership of the Committee for Mental Health Promotion. They were offered, however, under the leadership of individual governorate directors. Therefore, it was of interest to include a mental health training program as part of a national entity's responsibilities. ${ }^{6,15}$ The Director of the Montréal WHO-PAHO CC for Research and Training in Mental Health suggested the use of the mhGAP-IG training due to his familiarity with the program, his knowledge of its wide implementation in LMICs, ${ }^{29,30}$ and its novel training aspects, such as role plays, videos, and tools (i.e. guides and evaluation components). ${ }^{28}$ Besides meeting practical needs in the country, the implementation of the $m h G A P-I G$ in Tunisia would also serve to expand the program's limited evidence in French-speaking nations ${ }^{37}$ : Tunisia, to our knowledge, is one of the first French-speaking nations to implement and evaluate a mental health training based on the $m h G A P-I G .^{29,38}$

In addition, members of the Ministry of Health informed our team of the country's research gaps in the field of mental health. These included: a portrait of PCPs' mental health competencies in the Greater Tunis area, to help inform training material and aspects of health policy; an understanding of contextual barriers preventing the attainment of desired mental health training results, never explored before in Tunisia; and a short- and long-term assessment of the impact of an implemented mental health training program on PCPs' competencies, also never before assessed. Our research team sought to address these gaps by developing specific research objectives with accompanying methodologies that were deemed feasible by members of the Ministry of Health, all the while building local research capacity. 6,35 A randomized controlled trial (RCT) was thus suggested by members of the Ministry of Health and the WHO office in Tunisia. It was also supported by the directors of the governorates of the Greater Tunis area. This methodology allowed us to invite public-sector PCPs working in the Greater Tunis area to the mental health training and randomize participants into two groups: Group $1(n=52)$ and Group $2(\mathrm{n}=60)$. Both groups participated in the mental health training program at different times, ensuring: 1) that all those interested would receive the training; 2 ) that the training's short-term impact on PCPs' mental health com- 


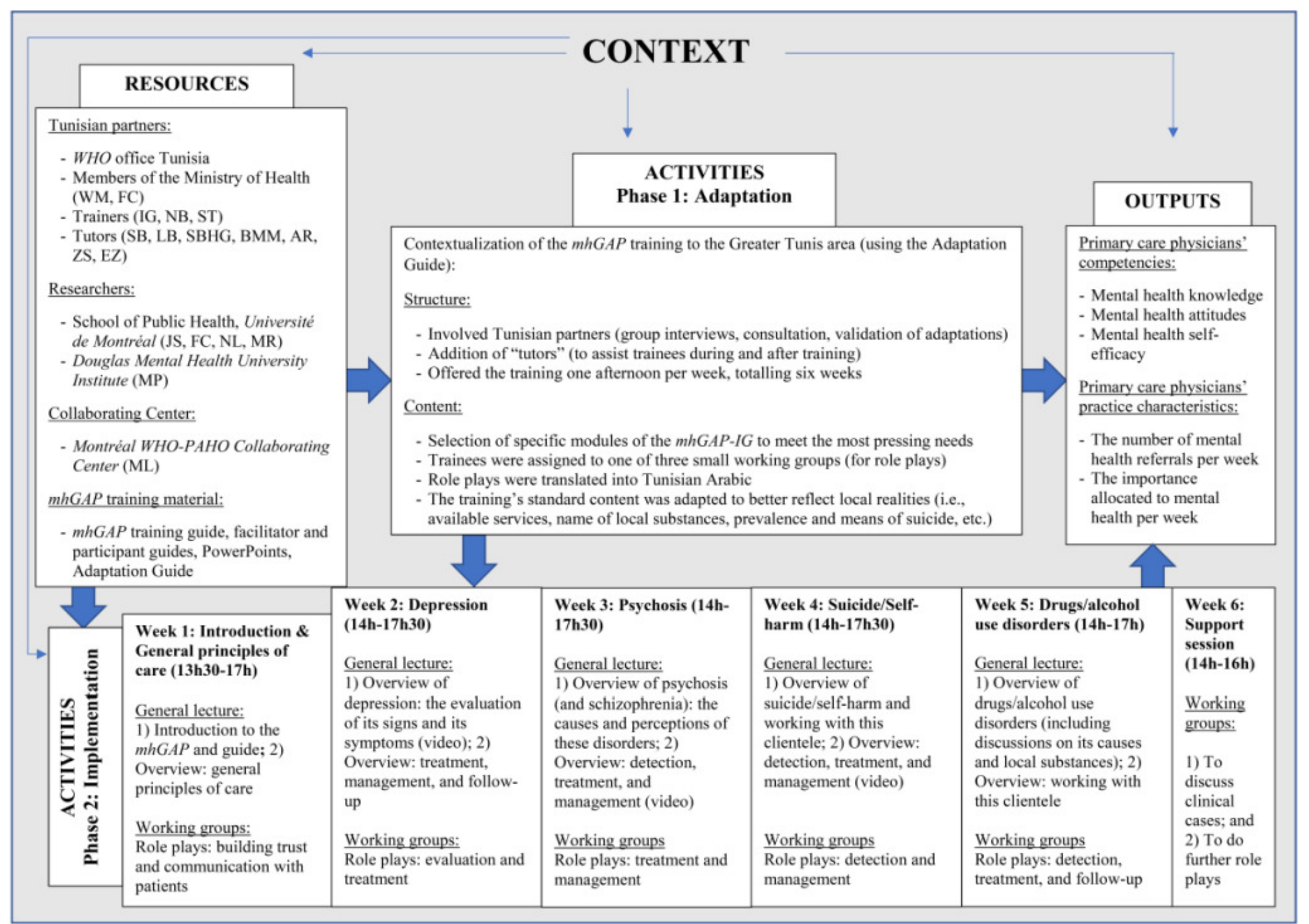

Figure 1. mhGAP-IG implementation model for the Greater Tunis area of Tunisia. 


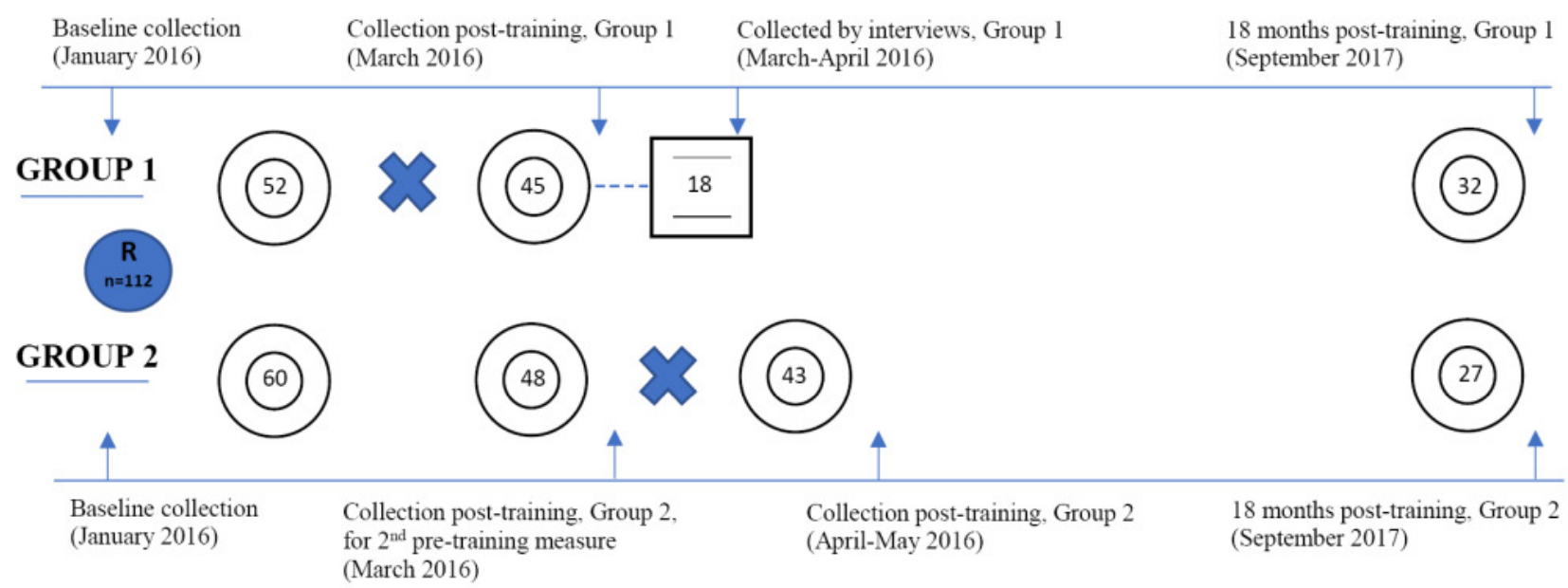

$\mathrm{R}=$ Randomization

$\mathrm{X}=$ Training

Figure 2. Evaluation of the $m h G A P-I G$ training in the Greater Tunis area of Tunisia.

petencies would be assessed in comparison to a control measure; and 3) that the training's long-term impact would be assessed by pooling both groups, increasing statistical power. Offering the training to both groups but at different times also encouraged the interviewing of PCPs who completed the first round of training in order to explore how contextual factors interacted with the implemented program to influence its expected outcomes. Of the 45 PCPs assigned to Group 1 who completed the training, 18 participated in individual or group interviews between March and April 2016. ${ }^{19}$ In addition, the RCT's pre-training questionnaires, administered to consenting PCPs prior to randomization $(n=112)$, helped us to paint a portrait of their mental health clinical practice and competencies. ${ }^{15}$ Figure $\underline{2}$ illustrates the adopted methodology to evaluate the training program in the Greater Tunis area of Tunisia.

The research objectives and methods discussed and developed in partnership are not only of interest to Tunisia but fit globally into the larger initiative of building research capacity in Global Mental Health. First, conducting an RCT where the intervention is offered to both groups of participants at different times responds to ethical questions raised around offering an intervention to one group over another despite the limited mental health resources in LMICs. 35 Second, to evaluate a mental health intervention such as a training program, RCTs and implementation analyses as complementary methodologies are encouraged to help generate practical (but local) knowledge for health systems. ${ }^{36}$ This practical and local evidence may influence important decisions regarding the intervention's scale-up within specific contexts. ${ }^{39,40}$ Third, results respond to the deficits in mental health evidence from LMICs. ${ }^{35}$ Specifically, while $90 \%$ of the global population live in LMICs, only between $3 \%$ and $6 \%$ of the mental health research published in highimpact journals comes from such countries. ${ }^{32}$
Some grants support partnerships for the development of research capacity, specifically for those partnerships within the field of Global Mental Health and with a vested interest in developing collaborations between income groups. ${ }^{35,41}$ These targeted opportunities are important considering the limited global health funding allocated specifically to Global Mental Health research, especially within LMICs. ${ }^{11,32,35,42}$ However, for this program, funding was obtained through organizations that support such partnerships more generally in the field of health: 1) Mitacs Globalink, ${ }^{43}$ an organization funded in part by the Government of Canada to create partnerships between academic institutions in order to better train students in global health research; and 2) the New Initiatives Funding of l'Institut de recherche en santé publique de l'Université de Montréal (IRSPUM), ${ }^{44}$ which supports new collaborations in order to develop research on topics currently under-represented at the School of Public Health at Université de Montréal. By applying to funding from initiatives beyond those centered solely on Global Mental Health research development, our aim was to increase the visibility of our project and our new collaboration, as well as the visibility of mental health in general, an under-represented discipline in global health. ${ }^{45}$

\section{LESSON 2: BENEFITTING FROM POLITICAL COMMITMENT TO MENTAL HEALTH}

Tunisia is a country politically invested in improving its mental health capacity, specifically by furthering the transition from institutional to community-based care. This vested interest has not only been seen in the drafting and adoption of the 2013 National Strategy for the Promotion of Mental Health ${ }^{6}$ but in important developments around this strategy. First, the Ministry of Health created the Committee for Mental Health Promotion to lead activities related to the strategy's implementation. Interestingly, the Ministry ap- 
pointed Dr. Wahid Melki as its Director, a chief psychiatrist at Hôpital Razi, the only operating mental health hospital in the country, ${ }^{6,17,18}$ but also a PCP by training. His early career as a PCP allowed him to truly grasp the challenges behind, and the necessity of, offering effective mental health care in primary care settings. Therefore, his vested interest has been to work on building PCPs' mental health competencies in primary care settings and to encourage the organizations in which they work to support this endeavor. Second, the Ministry of Health revamped the university curricula by drafting and passing a decree for the inclusion of a mandatory two-month mental health internship in postgraduate medical school that had previously been optional for future family physicians. ${ }^{46}$ The first graduating class under the new curricula is planned for 2019.

The drafting of the mental health strategy and Tunisia's interest in further developing PCPs' mental health competencies were not independent of contextual events but aligned with a process to involve Tunisian citizens in identifying potential tracks for country-wide health system reform. ${ }^{10}$ Such a process was locally known as le dialogue sociétal ["the societal dialogue"], where Tunisian citizens participated in focus groups to identify health care system challenges. ${ }^{10}$ After verbatim analysis, eight reform tracks were established, one of which was to strengthen health system capacity by creating proximity health services. ${ }^{10}$ This reorganization aimed to: 1 ) promote the use of multidisciplinary teams in primary care settings; 2 ) valorize general medical practice; and 3) further equip PCPs in effective patient management. This reform track was also discussed extensively as a way of meeting the untreated mental health needs in Tunisia, ${ }^{6}$ specifically by developing an already existent resource engaged in mental health care but with apparent deficits (i.e. PCPs). ${ }^{6,14-16}$

Our program worked amidst this political enthusiasm, or what the field of Global Mental Health calls "political commitment to mental health system development" (ie, "the organized intentions and actions of key decision-makers in a society, especially political leaders, to respond effectively to the mental health needs of the population" ${ }^{47}$ ). "Special attention" to mental health capacity-building in the country thus offered a unique way to forge and foster partnerships with a communal goal: the training of already graduated PCPs in effective mental health care using the $m h G A P-I G^{23}$ and the program's evaluation. Given this momentum and the mental health champions among our partners (ie, members of the Ministry of Health), funding for the implementation of the $m h G A P-I G$ training was covered by the WHO office in Tunisia, fostering local ownership of its implementation. In addition, findings show that benefitting from this political commitment to mental health could increase the use of research in policy by creating "a receptive policy environment [for] the "right research at the right time"”. ${ }^{8}$

\section{LESSON 3: PILOTING THE PROGRAM TO “BUILD BACK BETTER”}

"Building back better" is a term used by the WHO for mental health care after emergencies. ${ }^{49}$ In this paper, we use the term to refer to suggested improvements after piloting an intervention.
Given widespread untreated mental health symptoms in LMICs, the Global Mental Health movement aims to scale up evidence-based mental health interventions, particularly those that are feasible and effective at promoting the integration of mental health within primary and communitybased settings. ${ }^{20-22}$ Scaling up is defined as "efforts to increase the impact of innovations successfully tested in pilot or experimental projects so as to benefit more people and to foster policy and programme development on a lasting basis". ${ }^{50}$ At the heart of this definition is the piloting of interventions within contexts that are considering innovation scaleup to better understand if they are feasible, effective, and sustainable.

Several steps were taken to pilot the $m h G A P-I G$ in Tunisia. First, given that the $W H O$ encourages the adaptation of the $m h G A P-I G$ training to local contexts before implementation, ${ }^{23,26}$ our team allocated four months (i.e. September 2015 to January 2016) to its adaptation to the local primary healthcare reality of the Greater Tunis area. Our team published the adaptation details, ${ }^{17}$ which filled a gap in the Global Mental Health literature, ${ }^{29}$ to facilitate replication and/or help other LMICs undergo such a process. In brief, the adaptation process ensured that: 1) training modules from the mhGAP-IG were chosen to meet pressing local needs and contextually adapted; 2) a training schedule was tailored to the availability of PCPs to encourage their participation; 3) a support network of "tutors" was developed (ie, PCPs well-versed in mental health care and in charge of continuing medical education in the Greater Tunis area) to help trainees during and after training, which was especially important given specialists' heavy time constraints; 4) role plays were translated into Tunisian Arabic to mirror real-world consultations; and 5) gaps in mental health services within primary and community-based settings were identified. ${ }^{17}$

Our team believes that a strength of the adaptation process during this pilot phase was the creation of a support network for trainees, using an already existing yet available resource (ie, "tutors") in primary care settings. Given the WHO's emphasis on ongoing supervision when offering the mhGAP-IG training, ${ }^{23,26}$ but also Tunisia's inability to mobilize mental health personnel to provide such support, our team developed a realistic way of supporting trainees that could be piloted and easily reproduced on an ongoing basis should the program be scaled up. Of note, mobilizing an already existing yet available resource to provide support to trainees was similarly highlighted as a major strength during one of the first $m h G A P-I G$ training demonstrations on clinical utility in Nigeria. ${ }^{51}$ It is worth noting, however, that our team had limited contact with "tutors" post-training. Therefore, despite their role as "tutors" to trained PCPs, it is difficult to know how they explicitly conducted their assigned tasks post-training and to what extent they had an influence on the training program's expected outcomes.

Second, the adapted training program was assessed using an RCT. Pilot results suggest that the adapted program can increase mental health knowledge and self-efficacy, while decreasing referrals and negative mental health attitudes among PCPs in the Greater Tunis area of Tunisia. However, our findings reveal no impact on the importance PCPs allocate to mental health practice. While the goal of our pilot 
trial was not to generalize results to all PCPs working in Tunisia, but rather to see whether the training program worked in the Greater Tunis area, these results do hint at possible outcomes should the training program be offered to public sector PCPs working in other areas of Tunisia who would agree to participate in a mental health training. Regardless, an RCT design is unable to provide a plausible explanation for these findings. Hence, qualitative methods become necessary to better understand the context in which the intervention was implemented. ${ }^{27,52-54}$ Implementation analysis is thus a priority in the Global Mental Health field, since it helps identify practical challenges that decisionmakers could address to further encourage the implementation of programs that support the use of non-specialists such as PCPs in mental health care and that promote the integration of mental health into primary care settings. ${ }^{20-22,27,52-54}$

Eighteen Tunisian trainees interviewed identified several barriers when describing contextual factors influencing the mhGAP-IG training's expected outcomes. ${ }^{19}$ These include: 1) structural factors (e.g. restrictions that challenge PCPs' prescription of certain medications, stigma against substance use and misuse, the political favoritism of physical illnesses, and the non-systematic implementation of continuing mental health training for PCPs); 2) organizational factors (e.g. logistical issues for the provision of care, such as the lack and uneven distribution of certain medications, and the difficulty of collaborating with medical personnel untrained in mental health care); 3) provider factors (e.g. PCPs' limited mental health experience and their need to be self-motivated to provide care to patients consulting for mental health issues); 4) patient factors (e.g. patients' often negative beliefs about the health system and healthcare professionals, as well as their limited motivation to seek care); and 5) innovation factors (e.g. limits to the clinical utility of the training curriculum and issues with scheduling, potentially explaining drop-out). Interestingly, some of the contextual factors highlighted by trainees also mirror the gaps identified during the adaptation process. ${ }^{17}$ These include: lack and uneven distribution of psychotropic medications across healthcare clinics in the Greater Tunis area, stigma against substance use and substance misuse, as well as deficits in continuing mental health training for PCPs. ${ }^{17}$

Besides potentially affecting the integration of mental health into primary and community-based settings and influencing PCPs' involvement in the field of mental health, such contextual barriers are important to consider for two additional reasons. First, they may potentially reproduce or perpetuate, over the long-term, certain gaps uncovered prior to training in PCPs' mental health knowledge (i.e. lower scores on content related to substance use disorders and suicide/self-harm), attitudes (i.e. beliefs about the dangerousness of people with mental health issues), and selfefficacy (i.e. lower scores on confidence in capabilities to detect, treat, and manage what PCPs deem more complex mental health conditions, such as substance use disorders, suicide/self-harm, and psychosis). ${ }^{15}$ Second, contextual factors, identified by 18 interviewed PCPs, might challenge the training program's scale-up to other regions of Tunisia. Our sample of interviewed PCPs consisted of those working in the public sector from one area of Tunisia. However, we believe that our findings are useful for informing program scale-up. Specifically, PCPs working in the public sector of the Greater Tunis area experience similar barriers to effective mental health care as in other Tunisian regions. Nonetheless, while considering scaling up such a training program, it would be useful to develop and implement initiatives to tackle contextual factors that may challenge the attainment of its expected results.

Another promising feat of piloting an intervention is the ability to test the feasibility of implementing its specific modules, research methodology, and tools. First, when preparatory work was in progress prior to the implementation of the $m h G A P$-based training in the Greater Tunis area, some of the program's crucial elements were unavailable to the research team. For example, while rates of anxiety disorders have increased since the 2010-2011 Tunisian Revolution and remain a concern, at the time of adaptation and pilot implementation, the accompanying training material (i.e. PowerPoints, facilitator guides, and participant guides) for the module on conditions related specifically to stress 55 was not available in the language in which medical training is provided. This unavailability was an implementation barrier to a much-needed module. ${ }^{17}$

Second, by tailoring the standard training content and program to local primary care realities of the Greater Tunis area of Tunisia, systemic gaps were uncovered in resources for mental health treatment suggested by the $m h G A P-I G .^{23}$ These include deficits in community-based mental health services for people living with mental illness, such as little investment in subsidized housing and the unavailability of supported housing and supported employment initiatives. ${ }^{17}$ In addition, while many standard modules of the $m h G A P-I G$ include therapeutic interventions as part of the management skills to be developed by trainees (i.e. behavioural activation, interpersonal therapy, cognitive-behavioural therapy, contingency management therapy, family counselling/therapy, interpersonal psychotherapy, and motivational enhancement therapy), trainings in such therapies in Tunisia are reserved for psychosocial care providers, such as psychologists or psychiatrists. ${ }^{17}$ As a result, psychotherapy is very rarely conducted by PCPs in Tunisia. These uncovered deficits may be addressed by the promotion of treatments that use resources currently available in Tunisia (albeit distributed unevenly across the country), namely psychotropic medications. This reality in Tunisia $^{6,17}$ and in other LMICs ${ }^{11}$ might challenge the WHO's vision of the $m h G A P-I G$ 's self-sufficiency as a package offering a diverse set of complementary and necessary interventions for mental illness. ${ }^{23,24,26}$

Last, one of the most surprising discoveries made during the pilot testing of the $m h G A P-I G$ in the Greater Tunis area was the number of challenges PCPs had when asked to report their mental health statistics. PCPs reported these by filling out a mental health practice questionnaire based on the Mental, Neurological and Substance Use Patient Visit Summary developed by the WHO to accompany the $m h G A P$ $I G$ training and included in the original research protocol. ${ }^{38}$ Interestingly, these mental health statistical issues were explained by a complementary methodology: trainees acknowledged that while the Tunisian Ministry of Health has been encouraging PCPs to record mental health statistics 
per primary healthcare clinic, they also experienced limited follow-up by administrators, which has consequently jeopardized the institutionalization of proper record-keeping. ${ }^{19}$ Information from trainees on such a challenging part of the methodology helped us gain a clear understanding of why it proved problematic. In addition, administering questionnaires allowed us to provide some measure of reliability for these scales, based on our sample from the Greater Tunis area. Interestingly, while the attitudes questionnaire used in our trial (i.e. the Mental Illness: Clinicians' Attitudes (MICA) Scale (version 4.0) ${ }^{15,56,57}$ ) had acceptable internal consistency in a previous study, ${ }^{56}$ it did not show results that were as promising in our sample. ${ }^{15}$

Our pilot results, generated by diverse and complementary methodologies, ${ }^{52,53}$ may thus be used to "build back better" should the program be scaled up. Insight from our pilot program may be used to improve: 1 ) the training program itself, by rendering it more clinically useful and relevant; 2) the implementation of the training program, by ensuring that material accompanying certain modules is available; 2) the research program, by brainstorming on the tools best suited to collect data; 3) the mental health system, by addressing gaps in available resources and organizational barriers to effective mental health care and collaboration; and 4) mental health policies, by addressing restrictions on PCPs' prescription abilities and stigma against substance use and misuse.

\section{LESSON 4: SHARING RESEARCH FINDINGS}

Priority for the sharing of results has traditionally been through peer-reviewed publications, written reports, and conference presentations. ${ }^{58,59}$ Such mediums are important for the sharing of findings in the field of Global Mental Health, especially given evidence of the limited representation of mental health at international global health conferences $^{60}$ and in the global health literature. ${ }^{32,35}$

To share findings from this program, our team aimed to: 1) produce several publications in both English and French, the medical language in Tunisia; 2) participate in various research conferences; and 3) further develop individual research capacities by encouraging the involvement of local collaborators in the writing and publication process. ${ }^{34}$ For example, many of our Tunisian collaborators contributed to literature reviews, especially sections pertaining to information about the Tunisian healthcare system, the results and discussion sections, and manuscript revisions, all to ensure that information adequately represented contextual realities. Such involvement also aimed to build research capacity in the country 6 and, more generally, in the $E M R$, the WHO region in which Tunisia is represented. Records show that research initiatives in the EMR are disproportionately low in comparison to the mental health burden. 12,61,62

Despite the sharing of research findings through more traditional mediums, knowledge-to-action gaps in the Global Mental Health field continue to persist. ${ }^{63}$ Therefore, discussions of strategies for ensuring greater knowledge uptake to improve mental health practices, services, and policies beyond traditional mediums are of international focus. ${ }^{64,65}$ Findings reveal that knowledge translation (KT) strategies, which aim to move beyond the diffusion of find- ings uniquely towards the promotion of exchanges on such findings with key stakeholder groups, 53,59 have been shown to be effective in improving mental health practices and policies. ${ }^{64}$ Our team attempted to uphold the principles of KT by relying on a feasible strategy: the organization of a dissemination session in Tunisia. The session regrouped PCPs who participated in the training program and accompanying research, trainer-psychiatrists, PCPs in charge of continuing medical education in the Greater Tunis area (ie, "tutors"), members of the Ministry of Health, members of the WHO office in Tunisia, and directors of the governorates of the Greater Tunis area, in order to provide opportunities for exchange on preliminary findings from the program. Besides feedback on findings, this session resulted in the creation of key recommendations on ways to further PCPs' involvement in mental health care, some of which were identified by the research, while others moved beyond it. Recommendations were regrouped into a report and sent to all trainees for additional comments prior to being used as a reference by the mental health champions in our team during discussions with the Ministry of Health on future mental health priorities for the country.

Interestingly, when discussing the dissemination session, one of our Tunisian partners shared: "In my years involved in mental health research, this is the first attempt to regroup study participants and share with them the preliminary findings they helped produce." This statement was shocking to many of our Canadian collaborators, seeing as KT "has been adopted in Canada because translation of research is embedded in the mandate of the Canadian Institutes of Health Research (the federal agency for the funding of health research)". ${ }^{59}$ Therefore, encouraging and facilitating a culture of KT in Global Mental Health research is of utmost importance. Such development may be facilitated through targeted grants-for example, the one our research team received to disseminate results in the country in which the findings were collected ${ }^{66}$-or by making $\mathrm{KT}$ strategies mandatory upon the receipt of grants that fund Global Mental Health research.

\section{CONCLUSION}

The adaptation, implementation, and evaluation of a program based on the mhGAP-IG (version 1.0) ${ }^{23}$ in the Greater Tunis area of Tunisia generated important lessons learned, supported by evidence in the field of Global Mental Health. Our hope is that such experiential knowledge may be of use to other countries interested in addressing untreated mental health symptoms by developing/adapting, implementing, and evaluating programs that aim to build: 1) non-specialists' mental health competencies; and 2) the capacity of health systems to further integrate mental health into primary care. Both endeavors are priorities in Global Mental Health.

\section{ACKNOWLEDGEMENTS}

The authors wish to acknowledge: 1) Dr. Guido Sabatinelli, former WHO representative in Tunisia, and Ann-Lise Guis- 
set, $\mathrm{PhD}$, for their support in the development of this program and technical support while JS was in Tunisia; 2) Dr. Sonda Trabelsi, trainer-psychiatrist, for her dedication to the program; and 3) the WHO office in Tunisia, for their administrative support while JS was in Tunisia. JS would like to personally thank Matthew Rettino for his editing services.

\section{ETHICS}

Research approval was obtained from the Universite de Montréal (Québec, Canada) (\#15-117-CERES-D) and Razi Hospital (Manouba, Tunisia).

\section{DISCLAIMER}

The views expressed in the submitted article are the authors' and not an official position of the authors' institutions or research funders.

\section{FUNDING}

Jessica Spagnolo is funded by Fonds de recherche du Québec - Santé (FRQS, project \#33774). Data collection was funded by Mitacs Globalink (research fellowship, \#IT06835). The overall program in which this paper is inscribed is funded by
Institut de recherche en santé publique de l'Université de Montréal (IRSPUM) - New Initiatives Grants.

\section{COMPETING INTERESTS}

Dr. Marc Laporta works for the Montréal World Health Organization (WHO)-Pan American Health Organization (PAHO) Collaborating Center for Research and Training in Mental Health (Douglas Mental Health University Institute). All other authors declare no conflicts of interests. The authors completed the Unified Competing Interest form at http://www.icmje.org/coi_disclosure.pdf (available upon request from the corresponding author), and declare no further conflicts of interest.

\section{CORRESPONDENCE TO:}

\section{Jessica Spagnolo}

School of Public Health, Institut de recherche en santé publique de l'Université de Montréal (IRSPUM)

7101 Park Ave, Montreal

Québec Canada, H3N 1X9

jessica.maria-violanda.spagnolo@umontreal.ca 


\section{REFERENCES}

1. The World Bank. Data for 'Lower middle income', 'Tunisia.' Published online 2017. Accessed June 17, 2018. https://data.worldbank.org/?locations=XN-TN

2. Honwana A. Youth and Revolution in Tunisia. Palgrave Macmillan; 2013.

3. Ben Khelil M, Gharbaoui M, Farhani F, et al. Impact of the Tunisian Revolution on homicide and suicide rates in Tunisia. Int J Public Health.

2016;61(9):995-1002. doi:10.1007/s00038-016-0834-8

4. Ben Khelil M, Zgarni A, Zaafrane M, et al. Suicide by self-immolation in Tunisia: a 10 year study (2005-2014). Burns. 2016;42(7):1593-1599. doi:10.101 6/j.burns.2016.04.019

5. Charara R, Forouzanfar M, Naghavi M, et al. The burden of mental disorders in the Eastern Mediterranean region, 1990-2013. PLOS ONE. 2017;12(1):e0169575. doi:10.1371/journal.pone.0169 $\underline{575}$

6. La Stratégie nationale de promotion de la santé mentale. Ministry of Health, Tunis. Published 2013. Accessed July 10, 2018. https://www.mindbank.info/it em/6277

7. MedSPAD Committee. A first glance at the situation in the Mediterranean region in relation to the prevalence of alcohol, tobacco and drug use among adolescents. Published 2015. Accessed June 27, 2018. https://rm.coe.int/2017-ppg-med-15-medspad-region al-report-eng/16808cbcc1

8. Ouanes S, Bouasker A, Ghachem R. Psychiatric disorders following the Tunisian revolution. J Ment Health. 2014;23(6):303-306. doi:10.3109/09638237.20 14.928401

9. World Health Organization. Building general practitioner capacity in Tunisia by implementing the mhGAP. Published 2016. Accessed August 27, 2018. ht tp://www.who.int/mental health/mhgap/mhgap tuni sia/en/

10. Comité technique du dialogue sociétal. Pour une meilleure santé en Tunisie: faisons le chemin ensemble. Published 2014. Accessed August 27, 2017. http://www.hiwarsaha.tn/upload/1409228805.pdf

11. World Health Organization. Mental health atlas 2017. Published 2018. Accessed August 27, 2017. htt p://www.who.int/mental_health/evidence/atlas/ment al_health_atlas_2017/en/
12. Rahman A. Mental disorders in the Eastern Mediterranean Region. Int J Public Health. 2018;63(S1):9-10. doi:10.1007/s00038-017-0986-1

13. Bruckner TA, Scheffler RM, Shen G, et al. The mental health workforce gap in low- and middleincome countries: a needs-based approach. Bull World Health Organ. 2011;89(3):184-194. doi:10.2471/blt.1 $\underline{0.082784}$

14. Hend E, Haifa Z, Ghada K, Majda C, Afif B. Attitude des médecins généralistes face à la schizophrénie. Tunis Med. 2012;90(6):446-451.

15. Spagnolo J, Champagne F, Leduc N, et al. Mental health knowledge, attitudes, and self-efficacy among primary care physicians working in the Greater Tunis area of Tunisia. Int J Ment Health Syst. 2018;12(1):63. doi:10.1186/s13033-018-0243-x

16. Ben Thabet J, Mâalej M, Khemakhem H, et al. The management of depressed patients by the Tunisian general practitioners: a critical trans-sectional study. Community Ment Health J. 2019;55(1):137-143. doi:1 0.1007/s10597-018-0335-8

17. Spagnolo J, Champagne F, Leduc N, et al. Tailoring a training based on the Mental Health Gap Action Programme (mhGAP) Intervention Guide (IG) to Tunisia: process and relevant adaptations. Glob Ment Health (Camb). 2018;5:e17. doi:10.1017/gmh.2018.8

18. World Health Organization, Ministry of Health. WHO-AIMS Report on Mental Health System in Tunisia. WHO-AIMS; 2008. Accessed August 8, 2018. http://w ww.who.int/mental health/tunisia who aims_repor t.pdf

19. Spagnolo J, Champagne F, Leduc N, et al. "We find what we look for, and we look for what we know": factors interacting with a mental health training program to influence its expected outcomes in Tunisia. BMC Public Health. 2018;18(1):1398. doi:10.1 186/s12889-018-6261-4

20. Kohn R, Saxena S, Levav I, Saraceno B. The treatment gap in mental health care. Bull World Health Organ. 2004;82:858-866.

21. World Health Organization. Mental Health Action Plan, 2013-2020. WHO; 2013. Accessed July 15, 2018. http://apps.who.int/iris/bitstream/10665/89966/1/ 
22. Wainberg ML, Scorza P, Shultz JM, et al. Challenges and opportunities in global mental health: a research-to-practical perspective. Curr Psychiatry Rep. 2017;19(5):28. doi:10.1007/s11920-01 7-0780-z

23. World Health Organization. MhGAP Intervention Guide for Mental, Neurological and Substance Use Disorders in Non-Specialized Health Settings (Version 1.0). WHO Press; 2010. Accessed July 22, 2018. htt p://apps.who.int/iris/bitstream/10665/44406/1/97892 41548069 eng.pdf

24. World Health Organization. MhGAP: Scaling up Care for Mental, Neurological, and Substance Use Disorders. WHO Press; 2008. Accessed January 10, 2019. https://apps.who.int/iris/bitstream/handle/1066 5/43809/9789241596206 eng.pdf;jsessionid $=5 \mathrm{~EB} 1 \mathrm{C} 2 \mathrm{~B}$ 97B1E2895CA79C6E44F383888? sequence $=1$

25. Cohen A, Patel V, Minas H. A brief history of global mental health. In: Patel V, Minas H, Cohen A, Prince M, eds. Global Mental Health: Principles and Practice. Oxford University Press; 2013. https://doi.or $\mathrm{g} / 10.1093 / \mathrm{med} / 9780199920181.001 .0001$

26. World Health Organization. MhGAP Intervention Guide for Mental, Neurological and Substance Use Disorders in Non-Specialized Health Settings (Version 2.0). WHO Press; 2016. Accessed August 22, 2018. htt p://apps.who.int/iris/bitstream/10665/250239/1/9789 241549790-eng.pdf

27. Halon C, Fekadu A, Patel V. Interventions for mental disorders. In: Patel V, Minas H, Cohen A, Prince MJ, eds. Global Mental Health: Principles and Practice. Oxford University Press.

28. World Health Organization. MhGAP Operations Manual. WHO Press; 2018. Accessed January 20, 2019. https://www.who.int/mental_health/mhgap/operatio ns_manual/en/

29. Keynejad RC, Dua T, Barbui C, Thornicroft G. WHO Mental Health Gap Action Programme (mhGAP) Intervention Guide: a systematic review of evidence from low and middle-income countries. Evid Based Mental Health. 2018;21(1):30-34. doi:10.1136/eb-201 7-102750

30. World Health Organization. MhGAP Newsletter. WHO Department of Mental Health and Substance Abuse; 2018. Accessed August 17, 2018. https://mailc hi.mp/who/who-mhgap-newsletter-april-2018? $\mathrm{e}=\mathrm{a} 3 \mathrm{f}$ 1907369

31. Corbin JH, Mittelmark MB. Partnership lessons from the Global Programme for Health Promotion Effectiveness: a case study. Health Promot Int. 2008;23(4):365-371. doi:10.1093/heapro/dan029
32. Thornicroft G, Cooper S, Van Bortel T, Kakuma R, Lund C. Capacity building in global mental health research. Harv Rev Psychiatry. 2012;20(1):13-24. doi:1 $0.3109 / 10673229.2012 .649117$

33. Soska T, Johnson BA, eds. University-Community Partnerships: Universities in Civic Engagement. The Harworth Social Work Practice Press; 2004.

34. Acharya B, Maru D, Schwarz R, et al. Partnerships in mental healthcare service delivery in low-resource settings: developing an innovative network in rural Nepal. Global Health. 2017;13(1). doi:10.1186/s1299 2-016-0226-0

35. Collins PY, Tomlinson M, Kakuma R, Awuba J, Minas H. Research priorities, capacity, and networks in global mental health. In: Patel V, Minas H, Cohen A, eds. Global Mental Health: Principles and Practice. Oxford University Press; 2013:425-449. https://doi.or $\mathrm{g} / 10.1093 / \mathrm{med} / 9780199920181.003 .0019$

36. Patton M. Qualitative Research and Evaluation Methods. 4th ed. Sage Publications; 2015.

37. World Health Organization. MhGAP Newsletter. WHO Department of Mental Health and Substance Abuse; 2013. Accessed August 27, 2018. https://ww w.who.int/mental health/mhgap/mhGAP_nl_Decemb er_2013.pdf?ua $=1$

38. Spagnolo J, Champagne F, Leduc N, et al. Building system capacity for the integration of mental health at the level of primary care in Tunisia: a study protocol in global mental health. BMC Health Serv Res. 2017;17(1):38. doi:10.1186/s12913-017-1992-y

39. Altman DG. Sustaining interventions in community systems: on the relationship between researchers and communities. Health Psychol. 1995;14(6):526-536. doi:10.1037/0278-6133.14.6.526

40. Thornicroft G, Patel V. The importance of trials for global mental health. In: Thornicroft G, Patel V, eds. Global Mental Health Trials. Oxford University Press; 2014.

41. Grand Challenges Canada. Global Mental Health. Grand Challenges; 2018. Accessed August 1, 2018. htt p://www.grandchallenges.ca/programs/global-menta 1-health/

42. Collins PY, Pringle BA. Building a global mental health research workforce: perspectives from the National Institute of Mental Health. Acad Psychiatry. 2016;40(4):723-726. doi:10.1007/s40596-015-0453-3

43. Mitacs Globalink. Mitacs newsroom. Mitacs. Published 2019. Accessed August 1, 2018. https://ww w.mitacs.ca/en/newsroom/media-kit/about-mitacs-gl obalink 
44. Institut de recherche en santé publique. Bourses et subventions. Université de Montreal; 2018. Accessed August 14, 2018. http://www.irspum.umontreal.ca/fr$\mathrm{ca} /$ recherche/boursesetsubventions.aspx

45. Marquez PV, Saxena S. Making mental health a global priority. Cerebrum. 2016;2016:cer-10-6.

46. Ministère de la santé publique. Décret $n$ 2011-4132 du 17 novembre 2011, fixant le cadre général du régime des études médicales habilitant à l'exercice de la médecine de famille et à la spécialisation en médecine. Journal officiel de la République Tunisienne. 2011;90:2701-2708. http://ww w.atds.org.tn/DECRETNOV2011.pdf

47. Caldas de Almeida J, Minas H, Cayetano C. In: Patel V, Minas H, Cohen A, Prince MJ, eds. Global Mental Health: Principles and Practice. Oxford University Press; 2014:450-468.

48. Campbell DM, Redman S, Jorm L, Cooke M, Zwi $\mathrm{AB}$, Rychetnik L. Increasing the use of evidence in health policy: practice and views of policy makers and researchers. Aust N Z Health Policy. 2009;6(1):21. do i:10.1186/1743-8462-6-21

49. World Health Organization. Building Back Better: Sustainable Mental Health Care after Emergencies. WHO Press; 2013. Accessed January 30, 2019. http s://apps.who.int/iris/bitstream/handle/10665/85377/9 789241564571 eng.pdf?sequence $=1$

50. Simmons R, Fajans P, Ghiron L. Scaling up Health Service Delivery: From Pilot Innovations to Policies and Programmes. WHO Press; 2007. Accessed September 20, 2018. https://www.who.int/immunization/hpv/del iver/scalingup_health_service_delivery_who_2007.pdf

51. Gureje O, Abdulmalik J, Kola L, Musa E, Yasamy MT, Adebayo K. Integrating mental health into primary care in Nigeria: report of a demonstration project using the mental health gap action programme intervention guide. BMC Health Serv Res. 2015;15(1):242. doi:10.1186/s12913-015-0911-3

52. Palinkas LA. Qualitative and mixed methods in mental health services and implementation research. J Clin Child Adolesc Psychol. 2014;43(6):851-861. doi:1 $\underline{0.1080 / 15374416.2014 .910791}$

53. Creswell JW, Clark VLP. Designing and Conducting Mixed Methods Research. 3rd ed. Sage Publications; 2018.
54. Champagne F, Brousselle A, Hartz A, Contandriopoulos AP, Denis JL. L'analyse de l'implantation. In: Brousselle A, Champagne F, Contandriopoulos AP, Hartz Z, eds. L'évaluation: concepts et méthodes. Les Presses de l'Université de Montréal; 2011:238-379. https://doi.org/10.4000/boo ks.pum.6284

55. World Health Organization, United Nations High Commissioner for Refugees. Assessment and Management of Conditions Specifically Related to Stress: MhGAP Intervention Guide Module (Version 1.0). WHO Press; 2013. Accessed November 23, 2018. https://apps.who.int/iris/bitstream/handle/10665/856 23/9789241505932_eng.pdf?sequence $=1$

56. Gabbidon J, Clement S, van Nieuwenhuizen A, et al. Mental illness: clinicians' attitudes (MICA) scale psychometric properties of a version for healthcare students and professionals. Psychiatry Res. 2013;206(1):81-87. doi:10.1016/j.psychres.2012.09.02 8

57. The Indigo Network. Stigma scales. INDIGO. Published 2018. Accessed February 25, 2018. http://w ww.indigo-group.org/stigma-scales/

58. Pablos-Mendez A, Shademani R. Knowledge translation in global health. J Contin Educ Health Prof. 2006;26(1):81-86. doi:10.1002/chp.54

59. Straus SE, Tetroe J, Graham I. Defining knowledge translation. Can Med Assoc J. 2009;181(3-4):165-168. doi:10.1503/cmaj.081229

60. Spagnolo J, Turcotte-Tremblay AM. Global mental health: under-represented at international global health conferences? Health Systems Global. Published 2006. Accessed August 28, 2018. http://heal thsystemsresearch.org/hsr2016/is-global-mental-heal th-under-represented-at-internationally-acclaimed-g lobal-health-conferences/

61. Alwan A, Saeed K. A new agenda for mental health in the Eastern Mediterranean Region. Easter Mediterr Health J. 2015;12(7):459-460. doi:10.26719/2 $\underline{015.21 .7 .459}$

62. Regan M, Gater R, Rahman A, Patel V. Mental health research: developing priorities and promoting its utilization to inform policies and services. Easter Mediterr Health J. 2015;12(7):517-521. doi:10.26719/2 015.21.7.517

63. Thornicroft G. Evidence-based mental health care and implementation science in low- and middleincome countries. Epidemiol Psychiatr Sci. 2012;21(3):241-244. doi:10.1017/s2045796012000261 
64. Goldner EM, Jeffries V, Bilsker D, Jenkins E, Menear M, Petermann L. Knowledge translation in mental health: a scoping review. Healthc Policy. 2011;7(2):83-98. doi:10.12927/hcpol.2011.22620

65. Goldner EM, Jenkins EK, Fischer B. A narrative review of recent developments in knowledge translation and implications for mental health care providers. Can J Psychiatry. 2014;59(3):160-169. doi:1 $\underline{0.1177 / 070674371405900308}$
66. Quebec Population Health Research Network. Funding opportunity: support for results dissemination activities abroad. QPHRN. Published 2018. Accessed July 25, 2018. https://santepop.qc.ca/e $\mathrm{n}$ /presentation-results-award 